\title{
Metal wear debris generation in primary total knee arthroplasty: is it an issue?
}

\author{
Jonas Kellens, Pieter Berger, Hilde Vandenneucker
}

From the KU Leuven University of Leuven, Leuven, Belgium

More durable total knee arthroplasties (TKAs) are needed, due to the rising life expectancy, the higher activity levels of patients and the growing concerns about aseptic loosening being caused by metal hypersensitivity. In response, different hypoallergenic metal coatings have been developed for TKAs. However, possible adverse effects of these different metals (cobalt-chromium-molybdenum, zirconium, titanium and tantalum) have been neglected. The aim was to summarize the local and systemic adverse effects (including metal hypersensitivity), survival ratios, patient-reported outcome measures (PROMs) and the plasma metal ion concentrations of the different TKA coatings. A literature search on PubMed and EMBASE was performed. In total, 15 studies were found eligible. Common adverse effects of TKA were infection, loosening, pain, instability and hypercoagulation disorders. Serious adverse effects related to TKA implants were not reported. The survival ratios and patient-reported outcome measures seem to confirm these good results. In contrast with chromium and cobalt, no significant differences were reported in the nickel, molybdenum and titanium concentrations. No significant differences between the hypoallergenic and standard TKA implants were found in terms of adverse effects, survival ratios and PROMs. A causal relationship between the common adverse effects and the different metals is unlikely. Due to the heterogeneity of the TKA implants used, no firm conclusions could be made. Further research with longer follow-up studies are needed to find possible adverse effects and differences. Thus far, the hypoallergenic implants seem to perform equal to the standard implants.

No benefits or funds were received in support of this study. None of the authors have a conflict of interest.
Keywords: knee; prosthesis; arthroplasty; metal; adverse effects.

\section{INTRODUCTION}

Total knee arthroplasty (TKA) is a well-accepted treatment for knee osteoarthritis and other end-stage knee disorders. Due to the rising life expectancy, the higher activity levels of patients and the growing concerns about aseptic loosening being caused by metal hypersensitivity, more durable TKAs will be needed to satisfy the higher demands of patients. The 10-year survival ratio of TKA is approximately $90 \%$ (1). One of the major causes for revision is aseptic loosening, caused by wear debris, mainly polyethylene (1-4). Wear debris is generated by biotribocorrosion, a process induced by mechanical

Jonas Kellens ${ }^{1}$,

Pieter Berger ${ }^{2}$,

- Hilde Vandenneucker ${ }^{3}$

${ }^{1}$ Medical student, KU Leuven University of Leuven, Leuven, Belgium.

${ }^{2}$ Medical doctor, Department of Orthopaedics, University Hospitals Leuven, Leuven, Belgium.

${ }^{3}$ Medical doctor, Professor, Department of Development and Regeneration, KU Leuven University of Leuven, Leuven, Belgium.

Correspondence : Jonas Kellens. Ommegangstraat 7, 8870 Izegem, Belgium.

Email : jonas.kellens@yahoo.com

- 2021, Acta Orthopædica Belgica. 
wear, which ruptures the passive film formed on the prosthesis surface and thus leaves the metallic surface exposed to corrosive media $(2,5)$. However, the effects of metal wear debris (metal particles and ions), which is around $1 / 10^{\text {th }}$ of the total wear debris, have been underestimated. ${ }^{4}$ Metal wear can cause local and systemic adverse effects (3,4,6-10).

Local adverse effects, such as periprosthetic osteolysis, can be induced by metal ions that disrupt the normal bone metabolism. This by activating macrophages and osteoclasts, while deactivating osteoblasts (1,2,4,11-14). Metal hypersensitivity which is prevalent in approximately $60 \%$ of the patients with poorly functioning TKAs, can be caused by metal wear debris $(15,16)$. Metal ions can form chelates with native proteins, acting as antigens that form class-II major histocompatibility complexes on antigen-presenting cells. These present the antigens to T-cells, in order to trigger a cytokine reaction with the recruitment and activation of other inflammatory cells and thus causing an inflammatory reaction $(2,7,8,13)$. The clinical presentation of this inflammatory reaction can be summarized in pain, swelling, epi-cutaneous rash (eczema/dermatitis), patient dissatisfaction and loss of function in the knee $(16,17)$. Besides, metal ion concentrations in the systemic blood flow can also be raised after TKA, while the adverse effects of these raised levels are still unclear $(2-4,18)$.

To prevent complications and to expand the longevity of the prostheses, major improvements have been made on prosthesis design, alignment techniques and materials used for TKAs (2). Cobaltchromium-molybdenum prostheses are still the most used, but new coatings and prostheses with titanium, zirconium and tantalum are being developed and already in use with promising results $(3,6,12,18-21)$. Therefore, this review aims to summarize the local and systemic adverse effects, survival ratios and patient reported outcomes measures (PROMs) of cobalt-chromium-molybdenum, zirconium, tantalum and titanium total knee prostheses in primary TKA. Secondary attention will be given to metal hypersensitivity and the plasma metal ion concentrations.

\section{MATERIALS AND METHODS}

In order to collect data about possible adverse effects, survival ratios, PROMs and changes in plasma metal ion concentration after primary TKA in humans, searches were performed on PubMed and EMBASE between the $14^{\text {th }}$ of march 2020 and the $2^{\text {nd }}$ of July 2020 (cf. appendix I and II).

First, the articles were screened by title and abstract. Further selection was performed according to the exclusion and inclusion criteria. No crossreference checks were performed.

Exclusion criteria were unicondylar knee arthroplasty, focal knee arthroplasty, revision arthroplasty, prostheses with screws, infection as primary outcome, in vitro studies, nonhuman in vivo studies, case reports, (systematic) reviews, non-English articles, inability to access the full article and articles older than 10 years. Nonhuman studies were excluded since major errata have occurred with perfect working prostheses in nonhuman studies and extrapolating data would decrease the quality of this review (22). Articles that focussed on infection as a primary outcome were excluded, because these are correlated with the use of antibiotics and therefore were of no interest to this review. Only articles from the last 10 years were included, so that only relevant articles about metal prostheses in TKA used nowadays were used.

Inclusion criteria were total knee arthroplasty, low or moderate risk of bias with the 'risk of bias in nonrandomized studies - of interventions (ROBINS-I) assessment tool'. Total knee arthroplasty was defined as bicondylar replacement of the femur and a tibial plateau insert, with or without patellar resurfacing.

The following data, if available, were extracted out of the articles that met the selection criteria: author, study type, mean follow-up time(s), prosthesis type (femur material - tibia material type of cement), number of knees in each group (control groups included), number of knees lost to follow-up (LTFU), local and systemic adverse effects or complications, survival ratios (revision for aseptic loosening, all-cause revision, worst-case scenario), metal hypersensitivity reactions, plasma metal ion concentrations, PROMs like Knee Society 
Score (KSS), Oxford Knee Score (OKS), Western Ontario and McMaster Universities Osteoarthritis Index (WOMAC) and Knee Injury and Osteoarthritis Outcome Score (KOOS) and significance (p-value). When data were not available it was marked with 'NA' in the tables or just completely left out of the specific table. Derived data (data extracted out of charts or calculated) were marked by ' ${ }^{\circ}$ ' in the tables.

A qualitative analysis was performed by reporting the results for each outcome in the tables. Significant differences reported by the articles were also reported in this review with their corresponding p-values, and marked in the tables by '*' or '**'. Data was summarized for each outcome between the studies and TKA groups.

\section{RESULTS}

The performed searches when checked for duplicates, identified 476 different articles. These were screened by title and abstract for relevance. A total of 89 different articles were left for further assessment. After exclusion based on the exclusion criteria, 19 articles were left to be checked to meet the inclusion criteria. In total, 15 articles were included for data extraction (Figure 1, Table I).

\section{Cobalt-chromium-molybdenum (CoCrMo) or standard TKA group:}

In total, four RCTs, one prospective cohort study and three retrospective cohort studies reported outcomes of CoCrMo TKAs (Table I). In seven of these studies, the CoCrMo prostheses were used as the standard in control groups, therefore they can be named as the standard or conventional TKA group $(12,23)$.

The prostheses were completely made of uncoated CoCrMo in five studies (Table I). Infection, stiffness and deep vein thrombosis (DVT) were some of the adverse effects (Table II) $(3,6,12,14)$. Aseptic loosening occurred in $4.5 \%$ of the standard TKAs according to an RCT, not significantly differing from the TiNbN TKA group (12). Two studies reported no adverse effects $(23,24)$. One retrospective cohort study reported perfect survival ratios (Table III) (23). At 5-year follow-up, a signi-

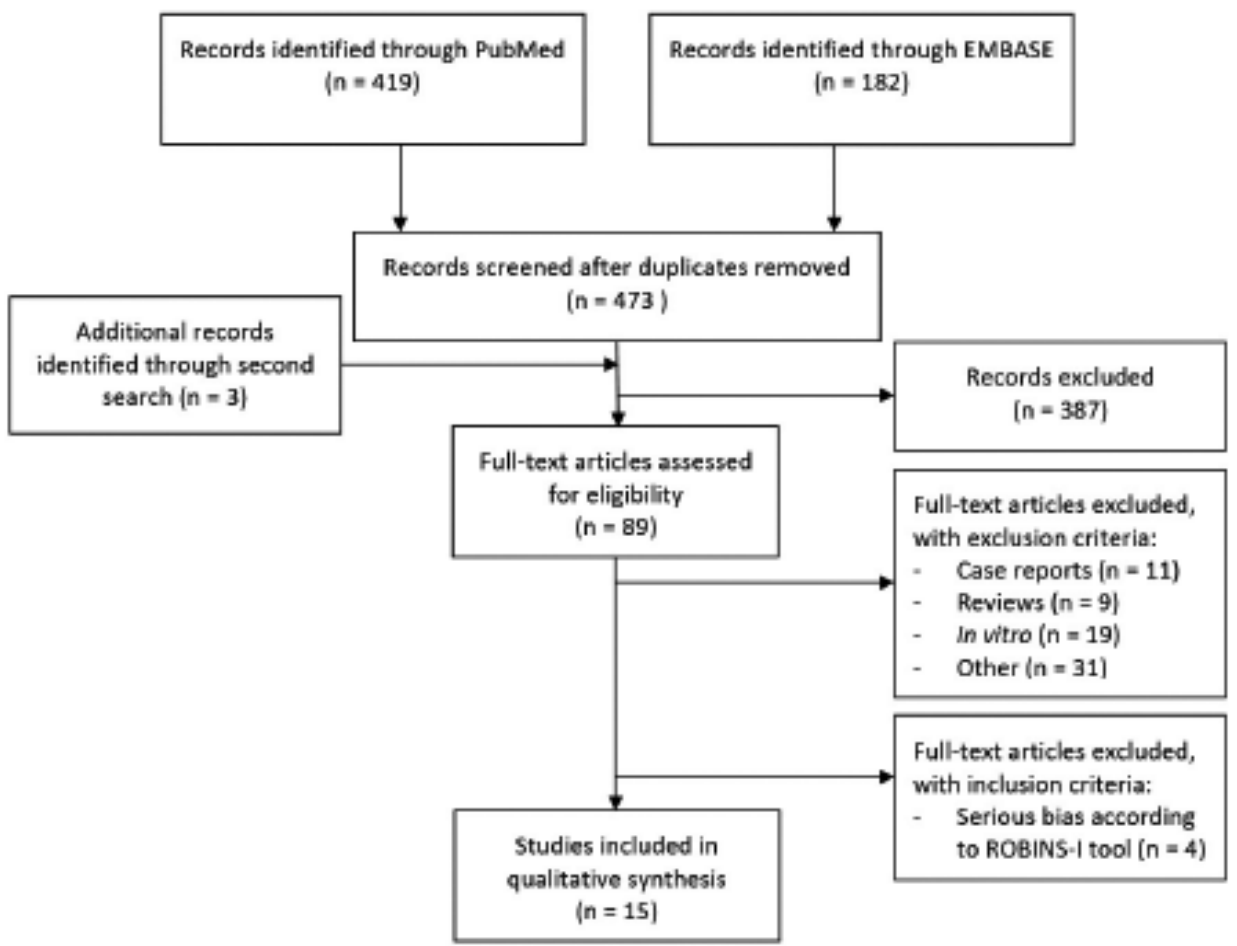

Figure 1. - PRISMA flow diagram of the article selection (34) $(\mathrm{n}=$ number of articles). 
Table I. - Information about the selected articles and prostheses used

\begin{tabular}{|c|c|c|c|c|c|}
\hline Author & $\begin{array}{l}\text { Type of } \\
\text { article }\end{array}$ & $\begin{array}{c}\text { Mean } \\
\text { follow-up } \\
\text { in years }\end{array}$ & $\begin{array}{c}\text { Number of } \\
\text { knees }\end{array}$ & $\begin{array}{c}\text { Femoral component - Tibial } \\
\text { component - Cement }\end{array}$ & Prosthesis type, brand \\
\hline Innocenti et al. ${ }^{26}$ & $\mathrm{CS}$ & 10 & 98 & $\begin{array}{l}\text { OxZr }- \text { TiAlV }(n=93) \text { or all- } \\
\text { PE }(n=5)^{19}-\text { Palacos }\end{array}$ & Genesis II Oxinium, Smith \& Nephew \\
\hline \multirow[t]{2}{*}{ Hui et al. ${ }^{20}$} & \multirow[t]{2}{*}{$\mathrm{RCT}$} & \multirow[t]{2}{*}{5} & 40 & OxZr - TiAlV - Simplex P & Genesis II Oxinium, Smith \& Nephew \\
\hline & & & 40 & CoCrMo - TiAlV - Simplex P & Genesis II, Smith \& Nephew \\
\hline Innocenti et al. ${ }^{11}$ & CS & 6.5 & 25 & $\mathrm{OxZr}-$ all-PE - NA & $\begin{array}{l}\text { Genesis II Oxinium, Smith \& Nephew, } \\
\text { All-Poly }\end{array}$ \\
\hline Papasoulis et al. ${ }^{25}$ & CS & 15 & 261 & OxZr - TiAlV ${ }^{19}-$ Palacos & Genesis II Oxinium, Smith \& Nephew \\
\hline \multirow[t]{2}{*}{ Vertullo et al. ${ }^{14}$} & \multirow[t]{2}{*}{$\mathrm{RC}$} & \multirow[t]{2}{*}{12} & 5,969 & $\mathrm{OxZr}-\mathrm{TiAlV}^{19}-\mathrm{NA}$ & Genesis II Oxinium, Smith \& Nephew \\
\hline & & & 11,608 & CoCrMo - TiAlV $^{19}-\mathrm{NA}$ & Genesis II, Smith \& Nephew \\
\hline Ahmed et al. ${ }^{27}$ & $\mathrm{CS}$ & 10 & 303 & OxZr - TiAlV ${ }^{19}-$ Simplex P & Genesis II Oxinium, Smith \& Nephew \\
\hline Glover et al. ${ }^{28}$ & $\mathrm{CS}$ & 8.5 & 103 & $\mathrm{OxZr}-$ all-PE - NA & Profix, Smith \& Nephew \\
\hline \multirow[t]{2}{*}{ Lützner et al. ${ }^{3}$} & \multirow[t]{2}{*}{$\mathrm{RCT}$} & \multirow[t]{2}{*}{1} & 61 & $\begin{array}{l}\text { Zirconium Nitride - } \\
\text { Zirconium Nitride - NA }\end{array}$ & Colombus Knee System AS, Aesculap \\
\hline & & & 59 & $\mathrm{CoCrMo}-\mathrm{CoCrMo}-\mathrm{NA}$ & Colombus Knee System, Aesculap \\
\hline Gerscovich et al. ${ }^{21}$ & $\mathrm{RC}$ & 10 & 108 & $\begin{array}{l}\text { High-flex fiber metal - } \\
\text { Tantalum - UC }\end{array}$ & NA \\
\hline \multirow[t]{2}{*}{ Postler et al. ${ }^{6}$} & \multirow[t]{2}{*}{ RCT } & \multirow[t]{2}{*}{3} & 59 & TiNbN - TiNbN - NA & BalanSys CR Knee System PVD, Mathys \\
\hline & & & 59 & CoCrMo - CoCrMo - NA & BalanSys CR Knee System, Mathys \\
\hline \multirow[t]{2}{*}{ Thienpont et al. ${ }^{23}$} & \multirow[t]{2}{*}{$\mathrm{RC}$} & \multirow[t]{2}{*}{2} & 40 & $\mathrm{TiNbN}-\mathrm{TiNbN}-\mathrm{NA}$ & Vanguard Biomet PVD \\
\hline & & & 80 & CoCrMo - CoCrMo - NA & Vanguard Biomet \\
\hline \multirow[t]{2}{*}{ Bergschmidt et al..$^{24}$} & \multirow[t]{2}{*}{$\mathrm{RC}$} & \multirow[t]{2}{*}{2} & 13 & $\begin{array}{l}\text { TiNbN }- \text { TiNbN }- \text { PMMA }(n= \\
\text { 8) or UC }(n=5)\end{array}$ & Genia TKR System PVD, ESKA implants \\
\hline & & & 12 & CoCrMo - CoCrMo - PMMA & Genia TKR System PVD, ESKA implants \\
\hline \multirow[t]{2}{*}{ Van Hove et al. ${ }^{12}$} & \multirow[t]{2}{*}{$\mathrm{RCT}$} & \multirow[t]{2}{*}{5} & 51 & $\mathrm{TiNbN}-\mathrm{TiNbN}-\mathrm{UC}$ & $\begin{array}{l}\text { CCI (now: ACS Basic), Implantcast } \\
\text { GmbH }\end{array}$ \\
\hline & & & 50 & CoCrMo - CoCrMo - UC & LCS Complete, DePuy Orthopedics Inc. \\
\hline Reiner et al. ${ }^{2}$ & $\mathrm{PC}$ & 1 & 25 & CoCrMo - TiAlV - NA & $\begin{array}{l}\text { P.F.C. SIGMA Total Knee System or TC3 } \\
\text { Knee System, DePuy Orthopedics Inc. }\end{array}$ \\
\hline \multirow[t]{2}{*}{ Wu et al..$^{29}$} & \multirow[t]{2}{*}{$\mathrm{RC}$} & \multirow[t]{2}{*}{14} & 1,092 & $\mathrm{NA}-\mathrm{NA}-\mathrm{NA}$ & NA \\
\hline & & & 8,096 & None & None \\
\hline
\end{tabular}

RCT: randomized controlled trial, RC: retrospective cohort study, PC: prospective cohort study, CS: cross-sectional study, OxZr: oxidized zirconium alloy, CoCrMo: cobalt-chromium-molybdenum, TiAlV: Titanium6\%-Aluminium4\%-Vanadium alloy, TiNbN: Titanium-niobium-nitride alloy, all-PE: all polyethylene, UC: uncemented or cementless, PMMA: polymethyl methacrylate, AS: advanced surfacing, PVD: physical vapour deposition, CCI: ceramic coated implant, LCS: low contact stress, NA: data not available.

ficant improvement was seen by one RCT in the knee and functional KSSs $(\mathrm{p}<0.001)$ (Table IV) (12). Two RCTs reported improvements in the 1-year postoperative OKS (Table V) (3,6). The WOMAC score at 2-year follow-up improved significantly in one study ( $\mathrm{p}=0.003$ ) (Table VI) (24). A KOOS of 80 at 2-year follow-up was reported (Table VII). (23) Two RCTs reported pre- and 1-year postoperative plasma metal ion concentrations $(3,6)$. The upperlimits preoperatively for cobalt (Co), chromium $(\mathrm{Cr})$, Molybdenum (Mo) and Nickel (Ni) were $2.02 \mu \mathrm{g} / \mathrm{L}, 1.55 \mu \mathrm{g} / \mathrm{L}, 3.06 \mu \mathrm{g} / \mathrm{L}$ and $2.53 \mu \mathrm{g} / \mathrm{L}$, respectively. One year postoperatively, the upperlimits for $\mathrm{Cr}$ and Mo were higher, $6.56 \mu \mathrm{g} / \mathrm{L}$ and $4.60 \mu \mathrm{g} / \mathrm{L}$ respectively, while the upper-limits for $\mathrm{Co}$ and Ni were lower than preoperatively (Table 
Table II. - Local and systemic adverse effects categorized by metal implant TKA group

\begin{tabular}{|c|c|c|c|}
\hline Author (mean FU) & $\mathbf{N}=$ & LTFU & Local and systemic adverse effects \\
\hline \multicolumn{4}{|c|}{ Cobalt-chromium-Molybdenum (CoCrMo) group } \\
\hline Van Hove et al. ${ }^{12}(5 y)$ & 50 & 6 & $\begin{array}{l}2 \text { tibial aseptic loosenings }(4.5 \%), 1 \text { deep infection, } 1 \text { patellar resurfacing, } 6 \\
\text { CPM, } 3 \text { MUA }\end{array}$ \\
\hline Postler et al. ${ }^{6}(3 y)$ & 59 & 11 & 1 infection, 4 MUA, 1 pulmonary embolism, 3 DVT \\
\hline Thienpont et al. ${ }^{23}(2 y)$ & 80 & 0 & None \\
\hline Bergschmidt et al. ${ }^{24}(2 y)$ & 12 & 0 & None \\
\hline Lützner et al. ${ }^{3}(1)$ & 59 & 3 & No implant related adverse events \\
\hline \multicolumn{4}{|c|}{ Titanium-aluminium-vanadium (TiAIV) group part of the CoCrMo group } \\
\hline Vertullo et al. ${ }^{14}(12 y)$ & 11608 & NA & $\begin{array}{l}\text { Infection, } 0.6 \% \text { loosening or lysis, pain, patellofemoral pain, instability (in } \\
\text { order of frequency) }\end{array}$ \\
\hline Hui et al. ${ }^{20}(5 y)$ & 40 & 3 & 2 patellar resurfacings \\
\hline Reiner et al. ${ }^{2}(1 y)$ & 25 & 1 & None \\
\hline \multicolumn{4}{|c|}{ Oxidized zirconium (OxZr) group } \\
\hline Glover et al. ${ }^{28}(8,5 y)$ & 103 & 0 & $\begin{array}{l}1 \text { traumatic tibial loosening, } 1 \text { tibial and femoral loosening, } 1 \text { infection, } 4 \text { joint } \\
\text { stiffness, } 1 \text { instability }\end{array}$ \\
\hline Innocenti et al. ${ }^{11}(6,5 y)$ & 25 & 0 & None \\
\hline \multicolumn{4}{|c|}{ Titanium-aluminium-vanadium (TiAlV) group part of the OxZr group } \\
\hline Papasoulis et al..$^{25}(15 y)$ & 261 & 59 & $\begin{array}{l}4 \text { aseptic loosening, } 5 \text { patellofemoral pain ( } 6 \text { patellar resurfacings, } 2 \text { poly- } \\
\text { ethylene exchanges), } 2 \text { traumatic peri-prosthetic fractures, } 1 \text { joint stiffness, } 1 \\
\text { superficial wound infection, } 21 \text { minor wound healing problems, } 8 \text { DVT, } 4 \text { MUA }\end{array}$ \\
\hline Vertullo et al..$^{14}(12 y)$ & 5969 & NA & $\begin{array}{l}1.1 \% \text { loosening or lysis, patellofemoral pain, infection, pain, instability (in } \\
\text { order of frequency) }\end{array}$ \\
\hline Ahmed et al. ${ }^{27}(10 y)$ & 303 & 85 & $\begin{array}{l}2 \text { infections, } 4 \text { anterior knee pain, } 2 \text { progressive osteoarthritis (6 patellar } \\
\text { resurfacings), } 1 \text { patellar tendon rupture, } 3 \text { pulmonary embolisms }\end{array}$ \\
\hline Innocenti et al. ${ }^{26}(10 y)$ & 98 & 10 & 2 femoral component loosening, 1 traumatic peri-prosthetic fracture \\
\hline Hui et al..$^{20}(5 y)$ & 40 & 3 & 2 patellar resurfacings \\
\hline \multicolumn{4}{|c|}{ Zirconium nitride (ZrN) group } \\
\hline Lützner et al. ${ }^{3}(1 y)$ & 61 & 1 & No implant related adverse events \\
\hline \multicolumn{4}{|l|}{ Tantalum (Ta) group } \\
\hline Gerscovich et al. ${ }^{21}$ (10y) & 108 & 50 & $\begin{array}{l}1,7 \% \text { aseptic loosening, } 1 \text { femur fracture, } 4 \text { extensor mechanism disruptions, } 2 \\
\text { tibial loosening, } 5 \text { unconfirmed revisions }\end{array}$ \\
\hline \multicolumn{4}{|c|}{ Titanium niobium nitride (TiNbN) group } \\
\hline Van Hove et al. ${ }^{12}(5 y)$ & 51 & 2 & $\begin{array}{l}2 \text { tibial aseptic loosening }(4 \%), 1 \text { layer spinout, } 8 \text { CPM, } 4 \text { MUA, } 2 \text { proximalisations } \\
\text { of the tibial tuberosity }\end{array}$ \\
\hline Postler et al. ${ }^{6}(3 y)$ & 59 & 4 & 1 traumatic wound dehiscence, 3 MUA, 1 lesion of the peroneal nerve, 1DVT \\
\hline Thienpont et al. ${ }^{23}(2 y)$ & 40 & 0 & 1 MUA \\
\hline Bergschmidt et al. ${ }^{24}(2 y)$ & 13 & 0 & 1 painful restriction, 1 traumatic femur fracture \\
\hline \multicolumn{4}{|l|}{ Unknown implant group } \\
\hline Wu et al. ${ }^{29}(14 y)$ & 1092 & NA & 291 eczema (incidence: 63.28 per 1000 person-years) \\
\hline \multicolumn{4}{|l|}{ Implant free group } \\
\hline Wu et al. ${ }^{29}(14 y)$ & 8096 & NA & 2162 eczema (incidence: 41.84 per 1000 person-years) \\
\hline
\end{tabular}

FU: follow-up, N: total number of knees, LTFU: number of knees lost to follow-up (including deaths), y: year(s), DVT: deep vein thrombosis, MUA: manipulation under anaesthesia, CPM: continuous passive motion, NA: data not available.

VIII). One RCT reported increasing median $\mathrm{Cr}$ concentrations, significantly higher in the standard TKA group compared to the TiNbN TKA group $(\mathrm{p}=0.012)(6)$.
The remaining three studies used a titaniumaluminium-vanadium (TiAlV) coated tibial base plate in combination with a femoral CoCrMo component (Table I). Loosening or lysis occurred in 0.6 
Table III. - Survival ratios of the different implant TKA groups

\begin{tabular}{|c|c|c|c|}
\hline Author (mean FU) & $\begin{array}{c}\text { Survival ratio of revision for } \\
\text { aseptic loosening }\end{array}$ & $\begin{array}{c}\text { Survival ratio for all-cause } \\
\text { revision }\end{array}$ & Worst-case survival ratio \\
\hline \multicolumn{4}{|c|}{ Cobalt-chromium-Molybdenum (CoCrMo) group } \\
\hline Thienpont et al. ${ }^{23}(2 y)$ & $100 \%$ & $100 \%$ & $100 \%$ \\
\hline \multicolumn{4}{|c|}{ Titanium-aluminium-vanadium (TiAlV) group part of the CoCrMo group } \\
\hline Vertullo et al. ${ }^{14}(12 y)$ & $99.4 \% \%^{\circ}$ & $95.2 \%(94.6 \%-95.8 \%)$ & NA \\
\hline Reiner et al. ${ }^{2}(1 y)$ & $100 \%$ & $100 \%{ }^{\circ}$ & $100 \% \%^{\circ}$ \\
\hline \multicolumn{4}{|c|}{ Oxidized zirconium (OxZr) group } \\
\hline Glover et al. ${ }^{28}(5 y \& 10 y)$ & $\begin{array}{c}100 \%{ }^{\circ} \\
\text { NA }\end{array}$ & $\begin{array}{l}94.1 \%(87.5 \%-97.5 \%) \\
95.4 \%(84.4 \%-99.7 \%)\end{array}$ & $\begin{array}{l}\text { NA } \\
\text { NA }\end{array}$ \\
\hline Innocenti et al. ${ }^{11}(6,5 y)$ & $100 \%$ & $100 \%$ & $100 \%$ \\
\hline \multicolumn{4}{|c|}{ Titanium-aluminium-vanadium (TiAIV) group part of the OxZr group } \\
\hline $\begin{array}{l}\text { Papasoulis et al. }{ }^{25}(10 y \& \\
15 y)\end{array}$ & $\begin{array}{l}98.4 \%(97.4 \%-99.9 \%) \\
98.4 \%(94.3 \%-99.9 \%)\end{array}$ & $\begin{array}{l}95.6 \%(92.8 \%-98.4 \%) \\
95.6 \%(92.7 \%-98.7 \%)\end{array}$ & $\begin{array}{l}88.1 \%(83.9 \%-92.1 \%) \\
85.1 \%(79.2 \%-96.2 \%)\end{array}$ \\
\hline Vertullo et al. ${ }^{14}(12 y)$ & $98.9 \% \%^{\circ}$ & $92.3 \%(90.5 \%-93.8 \%)$ & NA \\
\hline Innocenti et al. ${ }^{26}(10 y)$ & $97.8 \%(92.2 \%-99.4 \%)$ & NA & $86.1 \%(81.1 \%-92.7 \%)$ \\
\hline Ahmed et al. ${ }^{27}(10 y)$ & $100 \%$ & $97.1 \%(95.1 \%-99.1 \%)$ & $95.8 \%(94 \%-99 \%)$ \\
\hline \multicolumn{4}{|l|}{ Tantalum (Ta) group } \\
\hline Gerscovich et al..$^{21}(10 y)$ & NA & $95 \%$ & NA \\
\hline \multicolumn{4}{|c|}{ Titanium niobium nitride (TiNbN) group } \\
\hline Thienpont et al. ${ }^{23}(2 y)$ & $100 \%$ & $97.5 \%{ }^{\circ}$ & $97.5 \%{ }^{\circ}$ \\
\hline
\end{tabular}

Values were expressed as mean survival ratios, in parentheses the $95 \%$ confidence-interval if known. FU: follow-up, y: year(s), NA: data not available, ${ }^{\circ}$ : data calculated.

Table IV. - Knee society scores (KSSs) for the different implant TKA groups

\begin{tabular}{|c|c|c|c|c|}
\hline \multirow[t]{2}{*}{ Author (mean FU) } & \multicolumn{2}{|c|}{ Preoperative } & \multicolumn{2}{|c|}{ Postoperative } \\
\hline & Knee KSS & Functional KSS & Knee KSS & Functional KSS \\
\hline \multicolumn{5}{|c|}{ Cobalt-chromium-Molybdenum (CoCrMo) group } \\
\hline Van Hove et al..$^{12}(1 y \& 5 y)$ & $55.1(13.5)$ & $62.1(14.1)$ & $85^{\circ} \& 90^{\circ} *$ & $75^{\circ} \& 7^{\circ *}$ \\
\hline Thienpont et al. ${ }^{23}(2 y)$ & NA & NA & $90(10)$ & NA \\
\hline Lützner et al. ${ }^{3}(1 y)$ & $42.7(16.5)$ & $50.5(12.4)$ & $82.9(13.3)$ & $72.5(15.3)$ \\
\hline \multicolumn{5}{|c|}{ Titanium-aluminium-vanadium (TiAlV) group part of the CoCrMo group } \\
\hline Hui et al. ${ }^{20}(2 y \& 5 y)$ & NA & NA & NA & $88 \& 92$ \\
\hline Reiner et al. ${ }^{2}(1 y)$ & $32.5(11.8)(13-55)$ & $41.7(14.2)(5-80)$ & $84.8 *(13.5)(30-100)$ & $78.6 *(21.1)(15-100)$ \\
\hline \multicolumn{5}{|l|}{ Oxidized zirconium (OxZr) group } \\
\hline Innocenti et al. ${ }^{11}(6,5 y)$ & $38(18-59)$ & $39(5-55)$ & $91(65-100)$ & $88(55-100)$ \\
\hline \multicolumn{5}{|c|}{ Titanium-aluminium-vanadium (TiAIV) group part of the $\mathrm{OxZr}$ group } \\
\hline Papasoulis et al. ${ }^{25}(2 y \& 10 y \& 15 y)$ & 33.9 & 50.4 & $93.1 * \& 84.2 * \& 72.0 *$ & $88.0 * \& 73.1 * \& 58.4^{*}$ \\
\hline Innocenti et al..$^{26}(10 y)$ & $36(13-57)$ & $37(0-55)$ & $84(64-100)$ & $83(55-100)$ \\
\hline Hui et al. ${ }^{20}(2 y \& 5 y)$ & NA & NA & NA & $89 \& 89$ \\
\hline \multicolumn{5}{|l|}{ Zirconium nitride ( $\mathrm{ZrN}$ ) group } \\
\hline Lützner et al..$^{3}(1 y)$ & $44.5(13.4)$ & $52.0(12.3)$ & $84.3(11.6)$ & $75.9(18.3)$ \\
\hline \multicolumn{5}{|l|}{ Tantalum (Ta) group } \\
\hline Gerscovich et al. ${ }^{21}(10 y)$ & 36 & 46 & $99.2(2.97)$ & $97.4(12.9)$ \\
\hline \multicolumn{5}{|c|}{ Titanium niobium nitride (TiNbN) group } \\
\hline Van Hove et al. ${ }^{12}(1 y \& 5 y)$ & $58.1(16.2)$ & $62.2(14.4)$ & $85^{\circ} \& 90^{\circ} *$ & $75^{\circ} \& 70^{\circ} *$ \\
\hline Thienpont et al..$^{23}(2 y)$ & NA & NA & $88(13)$ & NA \\
\hline
\end{tabular}

Values were expressed as mean, in parentheses standard deviation and/or range. FU: follow-up, y: year(s), NA: data not available, ${ }^{\circ}$ : data extracted out of charts, *: significant $\mathrm{p}$-value $(\mathrm{p}<0.05)$. 
Table V. - Oxford Knee Scores (OKSs) for the different metal implant TKA groups

\begin{tabular}{|c|c|c|c|c|c|c|}
\hline \multirow[t]{2}{*}{ Author (mean FU) } & \multirow[t]{2}{*}{ Preoperative OKS } & \multicolumn{5}{|c|}{ Postoperative OKS } \\
\hline & & $1 \mathbf{y}$ & $2 \mathbf{y}$ & $3 \mathbf{y}$ & $10 y$ & $15 y$ \\
\hline \multicolumn{7}{|c|}{ Cobalt-chromium-Molybdenum (CoCrMo) group } \\
\hline Postler et al. ${ }^{6}$ & $21.0(7.0)$ & $37.6(7.4)$ & NA & $36.8(8.1)$ & NA & NA \\
\hline Lützner et al. ${ }^{3}$ & $21.8(7.4)$ & $37.5(7.8)$ & NA & NA & NA & NA \\
\hline \multicolumn{7}{|c|}{ Titanium-aluminium-vanadium (TiAIV) group part of the CoCrMo group } \\
\hline Reiner et al. ${ }^{2}$ & $18.9(6.9)(9-34)$ & $39.7(8.5)(5-40)^{*}$ & NA & NA & NA & NA \\
\hline \multicolumn{7}{|c|}{ Titanium-aluminium-vanadium (TiAIV) group part of the Oxidized zirconium (OxZr) group } \\
\hline Papasoulis et al. ${ }^{25}$ & 15.2 & NA & $42.0^{*}$ & NA & $37.4 *$ & $27.4 *$ \\
\hline \multicolumn{7}{|c|}{ Zirconium nitride (ZrN) group } \\
\hline Lützner et al. ${ }^{3}$ & $21.4(6.3)$ & $37.5(7.8)$ & NA & NA & NA & NA \\
\hline \multicolumn{7}{|c|}{ Titanium niobium nitride (TiNbN) group } \\
\hline Postler et al. ${ }^{6}$ & $20.9(6.1)$ & $37.9(7.4)$ & NA & $38.0(9.1)$ & NA & NA \\
\hline
\end{tabular}

Values were expressed as mean, in parentheses the standard deviation and/or range. FU: follow-up, y: year(s), NA: data not available, *: significant with $\mathrm{p}<0.05$.

Table VI — Western Ontario and McMaster Universities Osteoarthritis Index (WOMAC) scores for the different metal implant TKA groups

\begin{tabular}{|c|c|c|c|c|c|c|}
\hline \multirow{2}{*}{\multicolumn{2}{|c|}{ Author }} & \multirow[t]{2}{*}{ Preoperative } & \multicolumn{4}{|c|}{ Postoperative } \\
\hline & & & 2 years & 5 years & 10 years & 15 years \\
\hline \multicolumn{7}{|c|}{ Cobalt-chromium-Molybdenum (CoCrMo) group } \\
\hline \multicolumn{2}{|c|}{ Bergschmidt et al. ${ }^{24}$} & $\circ 55.7(9.9)$ & ${ }^{\circ} 26.5(31.0) *$ & $N A$ & $N A$ & $N A$ \\
\hline \multicolumn{7}{|c|}{ Oxidized zirconium (OxZr) group } \\
\hline Glover et al. ${ }^{28}$ & & 53 & NA & $29^{\wedge}$ & NA & NA \\
\hline \multicolumn{7}{|c|}{ Titanium-aluminium-vanadium (TiAIV) group part of the OxZr group } \\
\hline \multicolumn{2}{|c|}{ Papasoulis et al. ${ }^{25}$} & 55.4 & $13.1^{*}$ & NA & $17.2^{*}$ & $31.9^{*}$ \\
\hline \multirow[t]{2}{*}{ Ahmed et al. ${ }^{27}$} & Non-revision group (212 knees) & ${ }^{\circ} 52(16-88)$ & NA & NA & ${ }^{\circ} 14.3(0-85)^{*}$ & NA \\
\hline & Revision group (6 knees) & ${ }^{\circ} 52(16-87)$ & NA & NA & ${ }^{\circ} 17(2-33)$ & NA \\
\hline \multicolumn{7}{|c|}{ Titanium niobium nitride (TiNbN) group } \\
\hline \multicolumn{2}{|c|}{ Bergschmidt et al. ${ }^{24}$} & ${ }^{\circ} 51.0(16.1)$ & $\circ 39.5(38.0) *$ & $N A$ & $N A$ & $N A$ \\
\hline
\end{tabular}

Values were expressed as mean (range). Values in italic were expressed as median (interquartile range). NA: data not available, $*$ : significant with $\mathrm{p}<0.05, \wedge$ : $\mathrm{p}$-value not available, ${ }^{\circ}$ : data calculated, interquartile range not adapted, $95 \%$ confidence-interval adapted.

$\%$ of the TKAs according to the largest study with a mean follow-up time of 12 years (14). Other adverse effects were infection, pain, instability, restricted range of motion and DVT (14). One prospective cohort study reported no adverse effects (Table II) and had a perfect 1-year mean survival ratio (2). A retrospective 5-year follow-up cohort study reported a mean survival ratio for aseptic loosening of 99.4 $\%$ and a mean survival ratio for all-cause revision of $95.2 \%$ (Table III) (14). A prospective cohort study reported significant 1 -year postoperative improvements in both KSSs and OKS (all p<0.001) (Table IV \& V) (2). An RCT reported functional
KSSs of 88 and 92 at 2 and 5 years respectively (Table IV) (20). They also reported the 2- and 5-year postoperative KOOS of 82.5 and 84.25, respectively (Table VII) (20). The WOMAC scores were not reported. One prospective cohort study measured significant increases in median plasma Co concentrations 6 weeks postoperatively $(p=0.035)$. These sustained at 1 -year follow-up $(\mathrm{p}<0.001)$. The median plasma $\mathrm{Cr}$, Mo and $\mathrm{Ti}$ concentrations did not increase significantly at 1 -year follow-up (table VIII) (2). 
Table VII. - Knee Injury and Osteoarthritis Outcome Scores (KOOSs) for the different metal implant TKA groups

\begin{tabular}{|c|c|c|c|c|c|c|}
\hline \multirow{2}{*}{\multicolumn{2}{|c|}{ Author }} & \multirow[t]{2}{*}{ Preoperative } & \multicolumn{4}{|c|}{ Postoperative } \\
\hline & & & 2 years & 5 years & 10 years & 15 years \\
\hline \multicolumn{7}{|c|}{ Cobalt-chromium-Molybdenum (CoCrMo) group } \\
\hline \multicolumn{2}{|c|}{ Thienpont et al. ${ }^{23}$} & NA & $80(20)$ & NA & NA & NA \\
\hline \multicolumn{7}{|c|}{ Titanium-aluminium-vanadium (TiAIV) group part of the CoCrMo group } \\
\hline \multicolumn{2}{|l|}{ Hui et al. ${ }^{20}$} & NA & $\begin{array}{l}{ }^{\circ} 82.5 \\
93-75-75-87\end{array}$ & $\begin{array}{l}84.25 \\
93-70-86-88\end{array}$ & NA & NA \\
\hline \multicolumn{7}{|c|}{ Titanium-aluminium-vanadium (TiAIV) group part of the Oxidized zirconium (OxZr) group } \\
\hline \multicolumn{2}{|c|}{ Papasoulis et al. ${ }^{25}$} & $\begin{array}{l}31.0 \\
30.6-18.3-33.2- \\
38.2-9.7\end{array}$ & \begin{tabular}{|l|}
$76.0 *$ \\
$86.3-80.2-77.9-$ \\
$83.3-31.3$ \\
\end{tabular} & NA & $\begin{array}{l}73.0 * \\
82.7-71.2-74.2- \\
80.1-29.5\end{array}$ & $\begin{array}{l}65.5^{*} \\
73.8-63.4-64.2- \\
70.5-15.7\end{array}$ \\
\hline \multirow[t]{2}{*}{ Ahmed et al. ${ }^{27}$} & $\begin{array}{l}\text { Non-revision group } \\
\text { (212 knees) }\end{array}$ & NA & NA & NA & $\begin{array}{l}81.55 \\
87.5-71.4-84.9- \\
82.4\end{array}$ & NA \\
\hline & $\begin{array}{l}\text { Revision group (6 } \\
\text { knees) }\end{array}$ & NA & NA & NA & $\begin{array}{l}84 \\
86-86-82-82\end{array}$ & NA \\
\hline \multicolumn{2}{|l|}{ Hui et al. ${ }^{20}$} & NA & $\begin{array}{l}{ }^{\circ} 83 \\
93-76-76-87\end{array}$ & $\begin{array}{l}{ }^{\circ} 82.25 \\
90-68-84-87\end{array}$ & NA & NA \\
\hline \multicolumn{7}{|c|}{ Titanium niobium nitride ( $\mathrm{TiNbN}$ ) group } \\
\hline \multicolumn{2}{|c|}{ Thienpont et al. ${ }^{23}$} & NA & $77(18)$ & NA & NA & NA \\
\hline
\end{tabular}

Values were expressed as mean (standard deviation). The upper row gives the total KOOS score, the lower row(s) give the KOOS scores for pain - quality of life - symptoms - activities of daily living (- sports). NA: data not available, ${ }^{\circ}:$ data calculated, *: significant p-value with $\mathrm{p}<0.05$.

Table VIII. - Metal ion concentrations for the different metal implant TKA groups.

\begin{tabular}{|c|c|c|c|c|c|c|c|c|c|c|}
\hline \multirow[t]{2}{*}{ Author } & \multicolumn{5}{|c|}{ Preoperative } & \multicolumn{5}{|c|}{1 year postoperative } \\
\hline & Cobalt & Chromium & Molybdenum & Nickel & Titanium & Cobalt & Chromium & Molybdenum & Nickel & Titanium \\
\hline \multicolumn{11}{|c|}{ Cobalt-chromium-Molybdenum (CoCrMo) group } \\
\hline $\begin{array}{l}\text { Lützner } \\
\text { et al. }{ }^{3}\end{array}$ & $\begin{array}{c}{ }^{\circ} 0.67 \\
(0-2.02)\end{array}$ & $\begin{array}{l}{ }^{\circ} 0.50 \\
(0-1.55)\end{array}$ & $\begin{array}{c}1.21 \\
(0-3.06)\end{array}$ & $\begin{array}{c}{ }^{\circ} 0.88 \\
(0-2.53)\end{array}$ & NA & $\begin{array}{c}{ }^{\circ} 0.50 \\
(0-1.60)\end{array}$ & $\begin{array}{c}{ }^{\circ} 0.35 \\
(0-1.13)\end{array}$ & $\begin{array}{c}{ }^{\circ} 0.97 \\
(0-3.70)\end{array}$ & $\begin{array}{c}{ }^{\circ} 0.62 \\
(0-1.42)\end{array}$ & NA \\
\hline $\begin{array}{l}\text { Postler } \\
\text { et al. }\end{array}$ & $\begin{array}{c}0.25 \\
(0.25-1.25)\end{array}$ & $\begin{array}{c}0.25 \\
(0.25-1.12)\end{array}$ & $\begin{array}{c}0.25 \\
(0.25-1.88)\end{array}$ & $\begin{array}{c}0,50 \\
(0.50-1.50)\end{array}$ & NA & $\begin{array}{c}0.25 \\
(0.25-0.82)\end{array}$ & $\begin{array}{c}1.26^{* *} \\
(0.25-6.56)\end{array}$ & $\begin{array}{c}0.25 \\
(0.25-4.60)\end{array}$ & $\begin{array}{c}0.50 \\
(0.50-1.27)\end{array}$ & NA \\
\hline \multicolumn{11}{|c|}{ Titanium aluminium vanadium (TiAIV) group part of the CoCrMo group } \\
\hline $\begin{array}{l}\text { Reiner } \\
\text { et al. }\end{array}$ & $\begin{array}{c}0.006 \\
(0.005-0.141) \\
\end{array}$ & $\begin{array}{c}0.251 \\
(0.052-1.297) \\
\end{array}$ & $\begin{array}{c}0.421 \\
(0.225-1.221)\end{array}$ & NA & $\begin{array}{c}1.077 \\
(0.006-4.517) \\
\end{array}$ & $\begin{array}{c}0.243^{*} \\
(0.122-0.615) \\
\end{array}$ & $\begin{array}{c}0.268 \\
(0-1.984) \\
\end{array}$ & $\begin{array}{c}0.456 \\
(0.212-1.827) \\
\end{array}$ & NA & $\begin{array}{c}1.837 \\
(0.598-4.28) \\
\end{array}$ \\
\hline \multicolumn{11}{|c|}{ Zirconium nitride $(\mathrm{ZrN})$ group } \\
\hline $\begin{array}{l}\text { Lützner } \\
\text { et al. }{ }^{3}\end{array}$ & $\begin{array}{c}0.53 \\
(0-1.36)\end{array}$ & $\begin{array}{c}0.54 \\
(0-2.04)\end{array}$ & $\begin{array}{c}1.29 \\
(0-5.74)\end{array}$ & $\begin{array}{c}0.69 \\
(0-1.74)\end{array}$ & NA & $\begin{array}{c}0.45 \\
(0-1.38)\end{array}$ & $\begin{array}{c}0.31 \\
(0-0.69)\end{array}$ & $\begin{array}{c}0.90 \\
(0-3.03)\end{array}$ & $\begin{array}{c}0.78 \\
(0-2.43)\end{array}$ & NA \\
\hline \multicolumn{11}{|c|}{ Titanium niobium nitride (TiNbN) group } \\
\hline $\begin{array}{l}\text { Postler } \\
\text { et al. }{ }^{6}\end{array}$ & $\begin{array}{c}0.25 \\
(0.25-0.85)\end{array}$ & $\begin{array}{c}0.25 \\
(0.25-0.98)\end{array}$ & $\begin{array}{c}0.25 \\
(0.25-1.65)\end{array}$ & $\begin{array}{c}0.50 \\
(0.50-1.69)\end{array}$ & NA & $\begin{array}{c}0.25 \\
(0.25-1.19)\end{array}$ & $\begin{array}{c}0.75 * * \\
(0.25-3.92)\end{array}$ & $\begin{array}{c}0.25 \\
(0.25-1.14)\end{array}$ & $\begin{array}{c}0.50 \\
(0.50-1.00)\end{array}$ & NA \\
\hline
\end{tabular}

Values were expressed as median (range) in $\mu \mathrm{g} / \mathrm{L}$. NA: data not available, ${ }^{\circ}$ : data calculated, $*$ : significant with $\mathrm{p}<0.05$, $* *$ : significant difference between the two marked concentrations. 


\section{Oxidized zirconium (OxZr) TKA group}

One RCT, five cross-sectional studies and one retrospective cohort study were selected. The femoral components were all coated with OxZr, while 5 tibial components were made of TiAlV and 2 of polyethylene (Table I). No subdivisions were made between these. Loosening or lysis occurred in $1.1 \%$ according to the largest study with a mean follow-up time of 12 years (14). That retrospective cohort study also found a significant higher risk of revision caused by loosening or lysis in the subgroup aged 75 years or older with OxZr TKAs $(p=0.033)$ (14). Three post-traumatic periprosthetic fractures and a patellar tendon rupture were also reported. (2527) A cross-sectional study with a mean follow-up time of 6.5 years reported no complications (11). The same adverse effects as reported by the standard TKAs were also seen (Table II) $(14,25,27,28)$. The mean survival ratios of revision for aseptic loosening, minimum 10 years postoperative, varied from 97.8 to $100 \%(11,26-28)$. The mean survival ratios for all-cause revision, minimum 10 years postoperative, varied from 92.3 to $97.1 \%(11,14)$. One cross-sectional study reported a perfect worstcase survival ratio at a mean follow-up time of 6.5 years (11). The worst-case survival ratios, minimum 10 years postoperative, varied from 85.1 to 95.8 $\%$ (Table III) $(25,27)$. The mean preoperative knee KSSs varied from 33.9 to 38 (11,25). The mean knee KSS rose significantly at 2-year follow-up to 93.1 $(p=0.01)$ in one cross-sectional study. It declined to 72 at 15 -year follow-up, still significantly better than preoperatively ( $p=0.01)$ (25). These scores were also the minimum and maximum reported mean postoperative knee KSSs. Two other studies also reported improvements in the mean knee KSSs $(11,26)$. The mean preoperative functional KSSs varied from 37 to 50.4 , which improved to a mean postoperative functional KSSs varying from 58.4 to 89 (Table IV) $(20,25,26)$. The RCT reported no difference at any timepoint for the functional KSS compared to the standard TKA group (20). Solely one cross-sectional study reported a significant increase at all time points for both $\operatorname{KSS}(p=0.01)$ (25). The same cross-sectional study reported the OKS, which also significantly improved at all time points $(p=0.01)$ (Table V). A plateau from 3 to 10 years postoperatively was seen, followed by a decline afterwards (25). Two studies reported significant improvements in WOMAC scores $(\mathrm{p}=0.01$ and $\mathrm{p}<0.001)$ (Table VI) $(25,27)$. The same plateau and decline in the WOMAC scores were seen by the same study. They also noted a significant postoperative improvement in the KOOS at all timepoints $(\mathrm{p}=0.01)(25)$. The 10-year postoperative total KOOS varied from 73 to 84 (Table VII) $(25,27)$. No studies were found that measured the plasma metal ion concentrations.

\section{Zirconium nitride (ZrN) TKA group}

One RCT was selected. The femoral and tibial components were both coated with $\mathrm{ZrN}$ (Table I). No implant-related adverse events were reported (Table II). Both KSSs and OKS improved considerably at 1-year follow-up, while not significantly differing from the standard TKA group (Table IV \& V). Significant increases in mean plasma metal ion concentrations at 1-year follow-up were not measured (Table VIII). A survival ratio, WOMAC score and KOOS were not reported (3).

\section{Tantalum (Ta) TKA group}

One retrospective cohort study was selected. The tibial components consisted of tantalum, while the femoral components consisted of high-flex fiber metal (Table I). Aseptic loosening occurred in 1 out of 58 knees $(1.7 \%)$ left for evaluation. One femur fracture and four extensor mechanism disruptions were also reported (Table II). The mean 10-year survival ratio for all-cause revision was $95 \%$ (Table III). The knee and functional KSS both improved 10 years postoperatively (Table IV). Other PROMs and plasma metal ion concentrations were not reported (21).

\section{Titanium-niobium-nitride (TiNbN) TKA group}

Two RCTs and two retrospective cohort studies were selected. The femoral and tibial components were all coated with $\mathrm{ZrNbN}$ (Table I). One RCT reported 2 tibial aseptic loosenings $(4 \%)$ and 2 
proximalisations of the tibial tuberosity due to patella baja (12), while the other RCT reported a peroneal nerve lesion (6). One of the retrospective cohort studies reported a post-traumatic femur fracture (Table II) (24). The mean survival ratio of revision for aseptic loosening at 2-year follow-up was $100 \%$, while the mean worst-case survival ratio was $97.5 \%$ at 2-year follow-up (Table III) (23). One $\mathrm{RCT}$ and one retrospective cohort study reported KSSs and found no differences with their standard TKA groups $(12,23)$. However, the RCT reported a significant increase in both KSSs at 5-year followup (both $\mathrm{p}<0.001$ ) (Table IV) (12). The other RCT reported an improvement in OKS at 1-year followup, which sustained during 3-year follow-up (Table V) (6). The WOMAC scores reported by a retrospective cohort study improved significantly at 2-year follow-up $(\mathrm{p}=0.028)$, but no difference was seen with the standard TKA group (Table VI) (24). The KOOS reported by the other retrospective cohort study at 2-year follow-up did also not differ from the standard TKA group (Table VII) (23). The median plasma metal ion concentrations did not increase after TKA, except for $\mathrm{Cr}$, which rose from $0.25 \mu \mathrm{g} / \mathrm{L}$ to $0.75 \mu \mathrm{g} / \mathrm{L}$ at 1 -year follow-up (Table VIII). However, as already mentioned, this rise was significantly smaller compared to the standard TKA group ( $\mathrm{p}=0.012)$ (6).

\section{Titanium-aluminium-vanadium (TiAlV) TKA group}

Six studies reported about TiAlV coated tibial components. The femoral components were either made of CoCrMo or OxZr (Table I). Therefore these studies were summarized above in the standard TKA group (3 studies) and the OxZr TKA group (5 studies).

\section{Metal hypersensitivity}

Two studies about metal hypersensitivity were selected. A retrospective cohort study compared 1,092 patients with a TKA to 8,096 patients without any joint replacements. They found a significant higher chance to develop eczema in the TKA group (hazard ratio of 1.45 with a $95 \%$ confidence interval from 1.28 to $1.64, \mathrm{p}<0.0001$ )(Table II) (29). The RCT already mentioned above in the ZrN TKA group, found 1 new doubtful hypersensitivity reaction to $\mathrm{Ni}$ and 1 new weak reaction to Co in the ZrN TKA group at 2-year follow-up with patch-testing (skin testing). A new doubtful hypersensitivity reaction to $\mathrm{Ni}$ in the standard TKA group was also found. Skin reactions such as eczema or other implant related problems around the knee were not observed (3).

\section{DISCUSSION}

This review did not find serious adverse effects related to TKA implants. Rare complications, such as metallosis and pseudotumors linked to extensive metal wear, were not reported $(2,30)$. However, one peroneal nerve lesion was reported, probably caused by a pre-existing diabetes polyneuropathy and valgus malalignment in that patient, instead of an adverse effect of the TiNbN implant (6). Some studies, mostly of short follow-up time and one study with a mean follow-up of 6.5 years, did not find any complication $(2,11,23,24)$. Aseptic loosening, which is the main cause for revision after 3-year followup, did occur in most of the studies. A maximum aseptic loosening of $4.5 \%$ was reported. (12) The most common adverse effects of TKA seem to be infection, loosening, persisting pain, instability, stiffness, DVT and pulmonary embolism. However, causality with metal wear is unlikely, since there seems to be no difference between the TKA groups.

An important note is that there was a selection bias as to who received a hypoallergenic implant, which were younger patients or patients with a metal hypersensitivity (3). This could negatively influence the outcomes for the hypoallergenic implants, since younger patients may have a higher risk of prosthesis infection and loosening $(14,28)$. Younger patients will also put higher stresses on the prostheses. To exclude the confounder age, an age-stratified matched-cohort study analysed its results in age-groups. They found a significant higher revision for loosening or lysis $(p=0.033)$ in the OxZr TKA group of 75 years or older compared to the standard TKA group, which is unexpected. No explanation could be given for this finding, although, they did not correct their significance level for multiple testing. They also found no difference 
in revision for any reason in the younger patients between the OxZr and standard TKA groups (14).

According to one of the studies, eczema was more likely to occur in the TKA group (hazard ratio $1.45 ; 95 \%$ confidence interval 1.28-1.64, $\mathrm{p}<0.0001)$ compared to people without a joint replacement (hazard ratio 1) (29). This can be caused by metal hypersensitivity (16). However, metal hypersensitivity does not always have to cause skin reactions or other complications. A study found 3 new metal hypersensitivity reactions with patchtesting after TKA without any adverse effects around the knee (3). It still remains unknown whether there is a relationship between patch-testing and deep tissue hypersensitivity reactions $(3,31)$.

Survival ratios showed excellent prosthesis survival, with mean worst-case survival ratios varying from 85.1 to $100 \%$ (Table 3). One of these $100 \%$ worst-case survival ratios was reported by a study with $\mathrm{OxZr}$ implants at a mean follow-up time of 6.5 years (11). The mean worst-case survival ratios at 10 -year follow-up varied from 88.1 to $95.8 \%$ $(25,27)$. This is consistent with literature that reports a revision rate of 10 to $15 \%$ at longer follow-up $(1,32)$. Mean survival ratios of revision for aseptic loosening varied from 98.4 to $100 \%$, however, some of these studies reporting perfect survival had only 1 or 2 years of follow-up, which could be too short to observe aseptic loosening $(2,11,23,25,27,28)$. The mean survival ratios for all-cause revision varied from 92.3 to $100 \%(2,11,14,23)$. These survival ratios indicate that all TKA groups had equal good results, even in younger patients or patients with metal allergy. This is also an indication that adverse effects caused by metal ions are small or unlikely.

The revision rates could be negatively affected by including studies that did not perform patellar resurfacing at their primary TKA procedures $(14,33)$. The need for patellar resurfacing after TKA can be caused by anterior knee pain and progressive osteoarthritis. However, the same study that corrected for the confounder age, also performed an analysis that corrected for patellar resurfacing. No higher revision rates were found, this in contrast to the knowledge that TKAs done without resurfacing of the patella have higher risks of revision (14).
No significant differences in clinical outcome scores (KSS, OKS, WOMAC score and KOOS) were reported between the TKA groups at any time point. It is relevant to compare these clinical outcome scores, even when not validated for TKA. They might be more objective and sensitive, and say more about the efficacy than survival ratios alone $(25,27)$. A postoperative trend of improvement in the clinical outcome scores was seen in all studies, but not always significant. The hypoallergenic implants did, thus far, not perform significantly different than the standard TKA implants in terms of clinical outcome scores.

All three studies that measured plasma metal ion concentrations, found no difference at 1-year followup for Mo, Ni and $\mathrm{Ti}(2,3,6)$. Therefore, the plasma concentrations of Ni did not change significantly, which is promising since the most frequent metal hypersensitivity is related to $\mathrm{Ni}$ (6). One study found a significantly more pronounced postoperative increase in $\mathrm{Cr}$ concentration by the standard TKA group compared to the TiNbN group $(\mathrm{p}=0.012)$. In 13 patients ( 3 TiNbN TKA and 10 standard TKA patients), $\mathrm{Cr}$ concentrations higher than $2 \mu \mathrm{g} / \mathrm{L}$ were measured, which is considered a potential critical concentration for metal-on-metal hip arthroplasties (6). A potential critical metal ion concentration for TKA was not found in the literature. Another study found a significant increase of the Co concentration at 1-year follow-up within the TiAlV group (part of the CoCrMo TKA group) ( $<<0.001)$, all below 1 $\mu \mathrm{g} / \mathrm{L}$ (2). There were no studies found to report the plasma metal ion concentrations of the OxZr and $\mathrm{Ta}$ TKA groups. Conclusions could not be drawn about the alterations in plasma metal ion concentrations after TKA due to the short follow-up time of these studies.

Well-functioning TKAs have metal-on-polyethylene articulations, which causes the release of metal wear to be steadily. Therefore, possible increases in plasma metal ion concentration could be seen at longer follow-up times. However, some studies reported consistent plasma metal ion concentrations during longer follow-up on wellfunctioning TKAs (2). This in contrast to metal-onmetal total hip arthroplasties, where the metal wear is much higher during the first year, the running-in 
phase $(3,4,6)$. The metallic surface area exposed to body fluids, plays also a role in metal ion release through corrosion. TKA failure could thus cause increased plasma metal ion concentrations $(2,3,6)$. Studies that measure plasma metal ion concentrations over longer follow-up periods are needed in order to see potential differences in plasma metal ion concentrations and potential adverse effects of metal wear.

Several limitations to this literature review should be noted. Firstly, the heterogeneity of the TKA implants makes comparisons and conclusions very hard. For example, different coatings for the femoral and tibial components. Hence, no subdivisions were made in the OxZr TKA group between the polyethylene and TiAlV tibial base, since their results seemed to be comparable. Secondly, potential confounders such as the prosthesis design, type of cement, kind of polyethylene insert, patellar resurfacing or not, pre- and postoperative protocol, alignment strategy and surgical approach, etc. were not taken into account. Thirdly, there was an inherent selection bias for the hypoallergenic prostheses that could not be ruled out in this review, as recognized earlier. Fourthly, not all outcomes were available in the selected studies, which also limits our ability to make conclusions. Fifthly, the selection of studies and quality assessment was only performed by the first author. Sixthly, the ROBINS-I assessment tool was used to assess the quality, which is not validated to assess the quality of RCTs.

Strengths of this review were that it was focussed on primary TKA, and thus was not complicated by adverse effects of revision surgery or unicondylar/ focal knee arthroplasty. The review was thus able to make conclusions about primary TKA. Secondly, it focussed on relevant metals used nowadays in TKA, which makes it relevant in the working field. Thirdly, the ROBINS-I assessment tool was used as a quality assessment tool for the studies, appropriate for most of the selected studies.

\section{CONCLUSION}

This review found no significant differences between hypoallergenic and standard TKA implants in primary TKA, in terms of adverse effects, survival ratios, PROMs. Thus, hypoallergenic implants seem to perform equal to the standard implants, and could thus be used as an alternative. However, the price of hypoallergenic implants is higher, therefore a cost-benefit analysis should still be made for each patient. In order to find possible adverse effects of metal wear debris and its production in TKA, further research is needed. Studies with longer follow-up are also needed in order to find possible advantages of hypoallergenic implants compared to standard TKA implants.

\section{REFERENCES}

1. Xu, H., Guo, C.-C., Gao, Z.-Y. et al. Micrometer-Sized Titanium Particles Induce Aseptic Loosening in Rabbit Knee. Biomed Res. Int. 2018, 5410875.

2. Reiner, T., Sorbi, R., Müller, M. et al. Blood Metal Ion Release After Primary Total Knee Arthroplasty: A Prospective Study. Orthop. Surg. 2020, 12(2).

3. Lützner, J. , Hartmann, A., Dinnebier, G., SpornraftRagaller, P., Hamann, C. \& Kirschner, S. Metal hypersensitivity and metal ion levels in patients with coated or uncoated total knee arthroplasty: a randomised controlled study. Int. Orthop. 2013, 37: 1925-1931.

4. Kretzer, J. P., Reinders, J., Sonntag, R. et al. Wear in total knee arthroplasty - just a question of polyethylene?: Metal ion release in total knee arthroplasty. Int. Orthop. 2014, 38: 335-340.

5. Stojanović, B., Bauer, C., Stotter, C. et al. Tribocorrosion of a CoCrMo alloy sliding against articular cartilage and the impact of metal ion release on chondrocytes. Acta Biomater. 2019, 94: 597-609.

6. Postler, A., Beyer, F., Lutzner, C., Tille, E. \& Lutzner, J. Similar outcome during short-term follow-up after coated and uncoated total knee arthroplasty: a randomized controlled study. Knee Surg. Sports Traumatol. Arthrosc. 2018, 26: 3459-3467.

7. Thomas, P., Hisgen, P., Kiefer, H. et al. Blood cytokine pattern and clinical outcome in knee arthroplasty patients: comparative analysis 5 years after standard versus 'hypoallergenic' surface coated prosthesis implantation. Acta Orthop. 2018, 89(6): 646-651.

8. Du, Z., Wang, S., Yue, B., Wang, Y. \& Wang, Y. Effects of wear particles of polyether-ether-ketone and cobalt-chromium-molybdenum on CD4- and CD8-T-cell responses. Oncotarget 2018, 9(13): 11197-11208.

9. Paulus, A. C., Ebinger, K., Cheng, X. et al. Local Biological Reactions and Pseudotumor-Like Tissue Formation in relation to Metal Wear in a Murine In Vivo Model. Biomed Res. Int. 2019, 3649838.

10. Bandyopadhyay, A., Shivaram, A., Isik, M., Avila, J. D., Dernell, W. S. \& Bose, S. Additively manufactured calcium 
phosphate reinforced CoCrMo alloy: Bio-tribological and biocompatibility evaluation for load-bearing implants. Addit. Manuf. 2019, 28: 312-324.

11. Innocenti, M., Carulli, C., Matassi, F., Carossino, A. M., Brandi, M. L. \& Civinini, R. Total knee arthroplasty in patients with hypersensitivity to metals. Int. Orthop. 2014, 38: 329-333.

12. van Hove, R. P., Brohet, R. M., van Royen, B. J. \& Nolte, P. A. No clinical benefit of titanium nitride coating in cementless mobile-bearing total knee arthroplasty. Knee Surg. Sports Traumatol. Arthrosc. 2015, 23: 1833-1840.

13. Deng, Z., Wang, Z., Jin, J. et al. SIRT1 protects osteoblasts against particle-induced inflammatory responses and apoptosis in aseptic prosthesis loosening. Acta Biomater. 2017, 49: 541-554.

14. Vertullo, C. J., Lewis, P. L., Graves, S., Kelly, L., Lorimer, M. \& Myers, P. Twelve-Year Outcomes of an Oxinium Total Knee Replacement Compared with the Same Cobalt-Chromium Design: An Analysis of 17,577 Prostheses from the Australian Orthopaedic Association National Joint Replacement Registry. J. Bone Joint Surg. Am. 2017, 99: 275-283.

15. Granchi, D.,Cenni, E., Tigani, D., Trisolino, G., Baldini, N. \& Giunti, A. Sensitivity to implant materials in patients with total knee arthroplasties. Biomaterials 2008, 29: 14941500.

16. Desai, M. M., Shah, K. A., Mohapatra, A. \& Patel, D. C. Prevalence of metal hypersensitivity in total knee replacement. J. Orthop. 2019, 16: 468-472.

17. Craig, R., Vlychou, M., McCarthy, C. L., Gibbons, C. L. M. H. \& Athanasou, N. A. Metal wear-induced pseudotumour following an endoprosthetic knee replacement for Ewing sarcoma. Skeletal Radiol. 2017, 46: 967974.

18. Puente Reyna, A. L., Fritz, B., Schwiesau, J. et al. Metal ion release barrier function and biotribological evaluation of a zirconium nitride multilayer coated knee implant under highly demanding activities wear simulation. J. Biomech. 2018, 79: 88-96.

19. Innocenti, M., Civinini, R., Carulli, C., Matassi, F. \& Villano, M. The 5-year results of an oxidized zirconium femoral component for TKA. Clin. Orthop. Relat. Res. 2010, 468: 1258-1263.

20. Hui, C., Salmon, L., Maeno, S., Roe, J., Walsh, W. \& Pinczewski, L. Five-year comparison of oxidized zirconium and cobalt-chromium femoral components in total knee arthroplasty: a randomized controlled trial. $J$. Bone Joint Surg. Am. 2011, 93: 624-630.

21. Gerscovich, D., Schwing, C. \& Unger, A. Long-term results of a porous tantalum monoblock tibia component: clinical and radiographic results at follow-up of 10 years. Arthroplast. Today 2017, 3: 192-196.
22. Willis-Owen, C. A., Keene, G. C. \& Oakeshott, R. D. Early metallosis-related failure after total knee replacement: a report of 15 cases. J. Bone Joint Surg. Br. 2011, 93-B(2): 205-209.

23. Thienpont, E. Titanium niobium nitride knee implants are not inferior to chrome cobalt components for primary total knee arthroplasty. Arch. Orthop. Trauma Surg. 2015, 135: 1749-1754.

24. Bergschmidt, P., Bader, R., Finze, S., Schulze, C., Kundt, G. \& Mittelmeier, W. Comparative Study of Clinical and Radiological Outcomes of Unconstrained Bicondylar Total Knee Endoprostheses with Anti-allergic Coating. Open Orthop. J. 2011, 5: 354-360.

25. Papasoulis, E. \& Karachalios, T. A 13- to 16-year clinical and radiological outcome study of the genesis II cruciate retaining total knee arthroplasty with an oxidised zirconium femoral component. Knee 2019, 26: 492-499.

26. Innocenti, M., Matassi, F., Carulli, C., Nistri, L. \& Civinini, R. Oxidized zirconium femoral component for TKA: a follow-up note of a previous report at a minimum of 10 years. Knee 2014, 21: 858-861.

27. Ahmed, I., Salmon, L. J., Waller, A., Watanabe, H., Roe, J. P. \& Pinczewski, L. A. Total knee arthroplasty with an oxidised zirconium femoral component: ten-year survivorship analysis. Bone Joint J. 2016, 98-B(1): 58-64.

28. Glover, A. W., Santini, A. J. A., Davidson, J. S. \& Pope, J. A. Mid- to long-term survivorship of oxidised zirconium total knee replacements performed in patients under 50years of age. The Knee 2018, 25: 617-622.

29. Wu, P.-Y., Muo, C.-H. \& Tsai, C.-H. Increased risk of eczema after joint replacement: A population-based retrospective cohort study. Medicine 2019, 98(45): e17914.

30. Ho, J., Mahajan, J., Taylor, M., Byers, A., Arauz, P. \& Kwon, Y.-M. et al. Metallosis in cemented titanium alloy total knee arthroplasty without apparent metal-on-metal articulation. The Knee 2018, 25: 728-731.

31. Pellengahr, C., Mayer, W., Maier, M. et al. Resurfacing knee arthroplasty in patients with allergic sensitivity to metals. Arch. Orthop. Trauma Surg. 2003, 123: 139-143.

32. Du, Z., Zhu, Z. \& Wang, Y. The degree of peri-implant osteolysis induced by PEEK, CoCrMo, and HXLPE wear particles: a study based on a porous Ti6Al4V implant in a rabbit model. J. Orthop. Surg. Res. 2018, 13(23).

33. Vanlommel, J., De Corte, R., Luyckx, J. P., Anderson, M., Labey, L. \& Bellemans, J. Articulation of Native Cartilage Against Different Femoral Component Materials. Oxidized Zirconium Damages Cartilage Less Than CobaltChrome. J. Arthroplasty 2017, 32: 256-262.

34. Moher, D., Liberati, A., Tetzlaff, J. \& Altman, D. G. Preferred reporting items for systematic reviews and metaanalyses: The PRISMA statement. PLoS Med. 2009, 6(7). 


\section{Appendix I. Search strategy for the different metals used in TKA}

These searches were performed $14^{\text {th }}$ of March and the $5^{\text {th }}$ of May.

\section{Cobalt-Chromium total knee prosthesis:}

Search strategy PubMed:

((()(((““Arthroplasty, Replacement, Knee”[Mesh] OR "Knee Prosthesis"[Mesh] OR knee-replacement*[tiab] OR (("Bone-Anchored Prosthesis"[Mesh] OR "Prostheses and Implants"[Mesh] OR prosthe*[tiab] OR implant*[tiab] OR "resurfacing device"[tiab]) AND ("Knee"[Mesh] OR "Knee Joint"[Mesh] OR knee [tiab])) OR knee-arthroplast*[tiab] OR kneereconstruction*[tiab] OR knee-joint-replacement*[tiab] OR artificial-knee[tiab])) AND (Wear[tiab] OR "Waste Products"[Mesh] OR particle*[tiab] OR residue*[tiab]))) AND ("Metals"[Mesh] OR metal*[tiab]))) AND "last 10 years"[PDat])) AND ((cobalt-chromium) OR (cobalt OR chromium) OR (Co-Cr) OR (Co OR Cr))

Filters: published in the last 10 years

\section{Search strategy EMBASE:}

(('knee arthroplasty'/exp OR 'knee prosthesis'/ $\exp$ OR 'knee resurfacing device'/exp OR 'knee arthroplast*':ti,ab,kw OR 'knee prosthesis':ti,ab,kw OR 'knee resurfacing device':ti,ab,kw OR 'knee reconstruction':ti,ab,kw OR 'knee joint replacement*':ti,ab,kw) AND 'knee replacement arthroplasty':ti,ab,kw OR 'knee replacement*':ti,ab,kw OR 'artificial knee':ti,ab,kw) AND ('wear debris'/ $\exp$ OR 'waste'/exp OR 'residue':ti,ab,kw OR 'wear debris':ti,ab,kw OR 'waste':ti,ab,kw OR 'particle*':ti,ab,kw) AND ('cobalt'/exp OR 'cobalt':ti,ab,kw OR '59co':ti,ab,kw OR 'chromium'/ exp OR 'chromium':ti,ab,kw OR ‘chrome’:ti,ab,kw OR 'cr':ti,ab,kw OR '52cr':ti,ab,kw OR 'cocr':ti,ab,kw OR 'co-cr':ti,ab,kw) AND [2010-2020]/py

Titanium total knee prosthesis:

\section{$\underline{\text { Search strategy PubMed: }}$}

((()((((“Arthroplasty, Replacement, Knee”[Mesh] OR "Knee Prosthesis"[Mesh] OR knee-replacement*[tiab] OR (("Bone-Anchored Prosthesis"[Mesh] OR "Prostheses and Implants"[Mesh] OR prosthe*[tiab]
OR implant*[tiab] OR "resurfacing device"[tiab]) AND ("Knee"[Mesh] OR "Knee Joint"[Mesh] OR knee [tiab])) OR knee-arthroplast*[tiab] OR kneereconstruction*[tiab] OR knee-joint-replacement*[tiab] OR artificial-knee[tiab])) AND (Wear[tiab] OR "Waste Products"[Mesh] OR particle*[tiab] OR residue*[tiab]))) AND ("Metals"[Mesh] OR metal*[tiab]))) AND "last 10 years"[PDat])) AND ((Titanium) OR (Ti))

Filters: published in the last 10 years

\section{Search strategy EMBASE:}

(('knee arthroplasty'/exp OR 'knee prosthesis'/ $\exp$ OR 'knee resurfacing device'/exp OR 'knee arthroplast*':ti,ab,kw OR 'knee prosthesis':ti,ab,kw OR 'knee resurfacing device':ti,ab,kw OR 'knee reconstruction':ti,ab,kw OR 'knee joint replacement*':ti,ab,kw) AND 'knee replacement arthroplasty':ti,ab,kw OR 'knee replacement*':ti,ab,kw OR 'artificial knee':ti,ab,kw) AND ('wear debris'/ exp OR 'waste'/exp OR 'residue':ti,ab,kw OR 'wear debris':ti,ab,kw OR 'waste':ti,ab,kw OR 'particle*':ti,ab,kw) AND ('titanium'/exp OR 'titanium':ti,ab,kw OR ‘ti':ti,ab,kw) AND [2010-2020]/ py

Zirconium total knee prosthesis:

\section{Search strategy PubMed:}

(((()(((“Arthroplasty, Replacement, Knee”[Mesh] OR "Knee Prosthesis"[Mesh] OR knee-replacement*[tiab] OR (("Bone-Anchored Prosthesis"[Mesh] OR "Prostheses and Implants"[Mesh] OR prosthe*[tiab] OR implant*[tiab] OR "resurfacing device"[tiab]) AND ("Knee"[Mesh] OR "Knee Joint"[Mesh] OR knee [tiab])) OR knee-arthroplast*[tiab] OR kneereconstruction*[tiab] OR knee-joint-replacement*[tiab] OR artificial-knee[tiab])) AND (Wear[tiab] OR "Waste Products"[Mesh] OR particle*[tiab] OR residue*[tiab]))) AND ("Metals"[Mesh] OR metal*[tiab]))) AND "last 10 years"[PDat])) AND ((Zirconium) OR (Zr))

Filters: published in the last 10 years

\section{Search strategy EMBASE:}

(('knee arthroplasty'/exp OR 'knee prosthesis'/ exp OR 'knee resurfacing device'/exp OR 'knee 
arthroplast*':ti,ab,kw OR 'knee prosthesis':ti,ab,kw OR 'knee resurfacing device':ti,ab,kw OR 'knee reconstruction':ti,ab,kw OR 'knee joint replacement*':ti,ab,kw) AND 'knee replacement arthroplasty':ti,ab,kw OR 'knee replacement*':ti,ab,kw OR 'artificial knee':ti,ab,kw) AND ('wear debris'/ exp OR 'waste'/exp OR 'residue':ti,ab,kw OR 'wear debris':ti,ab,kw OR 'waste':ti,ab,kw OR 'particle*':ti,ab,kw) AND ('zirconium'/exp OR 'zirconium':ti,ab,kw OR 'zr':ti,ab,kw) AND [2010$2020] / p y$

\section{Tantalum total knee prosthesis:}

Search strategy PubMed:

(()((((““Arthroplasty, Replacement, Knee”[Mesh] OR "Knee Prosthesis"[Mesh] OR knee-replacement*[tiab] OR (("Bone-Anchored Prosthesis"[Mesh] OR "Prostheses and Implants"[Mesh] OR prosthe*[tiab] OR implant*[tiab] OR "resurfacing device"[tiab]) AND ("Knee"[Mesh] OR "Knee Joint"[Mesh] OR knee [tiab])) OR knee-arthroplast*[tiab] OR knee- reconstruction*[tiab] OR knee-joint-replacement*[tiab] OR artificial-knee[tiab])) AND (Wear[tiab] OR "Waste Products"[Mesh] OR particle*[tiab] OR residue*[tiab]))) AND ("Metals"[Mesh] OR metal*[tiab]))) AND "last 10 years"[PDat])) AND ((tantalum) OR (trabecular metal))

Filters: published in the last 10 years

\section{Search strategy EMBASE:}

(('knee arthroplasty'/exp OR 'knee prosthesis'/ exp OR 'knee resurfacing device'/exp OR 'knee arthroplast*':ti,ab,kw OR 'knee prosthesis':ti,ab,kw OR 'knee resurfacing device':ti,ab,kw OR 'knee reconstruction':ti,ab,kw OR 'knee joint replacement*':ti,ab,kw) AND 'knee replacement arthroplasty':ti,ab,kw OR 'knee replacement*':ti,ab,kw OR 'artificial knee':ti,ab,kw) AND ('wear debris'/ exp OR 'waste'/exp OR 'residue':ti,ab,kw OR 'wear debris':ti,ab,kw OR 'waste':ti,ab,kw OR 'particle*':ti,ab,kw) AND ('tantalum'/exp OR 'tantalum':ti,ab,kw OR 'ta':ti,ab,kw) AND [2010-2020]/ py

\section{Appendix II: Search strategy for metal hypersensitivity in TKA}

These searches were performed the $14^{\text {th }}$ of March and the $2^{\text {nd }}$ of July.

\section{Search strategy PubMed:}

(((((“Hypersensitivity”[Mesh]) OR (“Allergy and Immunology”[Mesh])) OR hypersensitivity[tiab]) OR allergy[tiab])) AND (((“Arthroplasty, Replacement, Knee"[Mesh] OR “Knee Prosthesis”[Mesh] OR kneereplacement*[tiab] OR (("Bone-Anchored Prosthesis"[Mesh] OR "Prostheses and Implants"[Mesh] OR prosthe*[tiab] OR implant*[tiab] OR "resurfacing device"[tiab]) AND ("Knee"[Mesh] OR "Knee Joint"[Mesh] OR knee [tiab])) OR knee-arthroplast*[tiab] OR knee-reconstruction*[tiab] OR knee-joint-replacement*[tiab] OR artificial-knee[tiab])))

\section{Search strategy EMBASE:}

(('knee arthroplasty'/exp OR 'knee prosthesis'/ $\exp$ OR 'knee resurfacing device'/exp OR 'knee arthroplast*':ti,ab,kw OR 'knee prosthesis':ti,ab,kw OR 'knee resurfacing device':ti,ab,kw OR 'knee reconstruction':ti,ab,kw OR 'knee joint replacement*':ti,ab,kw) AND 'knee replacement arthroplasty':ti,ab,kw OR 'knee replacement*':ti,ab,kw OR 'artificial knee':ti,ab,kw) AND ('allergy':ti,ab,kw OR 'hypersensitivity':ti,ab,kw OR 'hypersensitivity'/exp OR 'allgergy') 